\title{
Integral Operators of Mixed Type of Nonlinear Integral Equation
}

\author{
Lamyaa H. Saadoon \\ College of Education \\ Department of mathematics
}

Received

24/09/2012
Accepted

14/11/2012

الملنص

في هذا البحث, ندرس بعض خواص المؤثرات التكاملية غير الخطية لنوع مختلط من المعادلات التكاملية بأنوية تعتمد على متغيرين. تم الحصول على النتائج المتعلقة بالاستمرارية

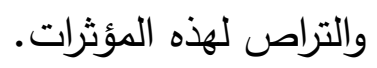

\section{$\underline{\text { Abstract }}$}

In this paper, we investigate some properties of nonlinear integral operators of mixed type of integral equations with kernels depending on two variables. The results concerning the continuity and compactness of these operators are obtained.

Key words: Nonlinear integral operators, function of bounded variation, Stieltjes- integral, Volterra - integral, Fredholm - integral, non decreasing function, compact operator.

\section{Introduction}

The theory of integral operators is an important part of nonlinear analysis and their applications to real world problems $[1,4,5]$.

The theory of integral equations in now well developed with the help of various tools of functional analysis, topology and fixed point theory.[ 6,7$]$

On the other hand many authors can meet several papers or books devoted to the study of integral operators of Fredholm, Volterra, Hammerstein and Urysohn type are used frequently when describing real-world problems $[4,8]$. It is interest to note that the integral operators mentioned above can be treated as special 
cases of integral operators of Stieltjes type with kernels depending on two variables $[9,10]$.

In this paper we will study integral operators mixed type of nonlinear of integral equations. These operators are defined with the help of Riemann-Stieltjes integral with kernels depending on two real variables.

We begin by proving a few results concerning the continuity, bounded variation, monotonicity and compactness of the mixed type of integral operators in the space of continuous functions. The results of such kind were obtained also in the papers [2,3]. Here, we generalize and improve these results.

\section{Notations, Definitions And Some Properties of The Stieltjes integral}

In this section, we collect a few auxiliary facts which will be needed in the sequel. At the beginning, we recall some basic concepts and results concerning functions of bounded variation and Stieltjes integral.

Definition (1) [7]: A real function $\mathrm{x}$ defined on the interval $[\mathrm{a}, \mathrm{b}]$ the symbol $V_{a}^{b} x$ denotes the variation of $\mathrm{x}$ on $[\mathrm{a}, \mathrm{b}]$, we say that $\mathrm{x}$ is of bounded variation whenever $V_{a}^{b} x$ is finite. If $u(t, s)=u:[a, b] \times[c, d] \rightarrow R$, then, we denote by $\underset{t=p}{V} u(t, s)$, the variation of the function $t \rightarrow u(t, s)$ on the interval $[p, q] \subset[a, b]$, where $\mathrm{s}$ is arbitrarily fixed in [c, d]. Similarly, we define the quantity $\stackrel{q}{V} u(t, s)$. We refer to [7] for the properties of functions of bounded variation.

Definition (2) [7]: If $x$ and $\varphi$ are two real bounded functions defined on the interval $[a, b]$, then, under some additional conditions, we can define the Stieltjes integral ( in the Riemann - Stieltjes sense),

$$
\int_{a}^{b} x(t) d \varphi(t)
$$

of the function $\mathrm{x}$ with respect to the function $\varphi$. In this case, we say that $\mathrm{x}$ is Stieltjes integrable on the interval $[a, b]$ with respect to $\varphi$. 
There are known several conditions guaranteeing the Stieltjes integrablility [7]. One of the most frequently used requires that $\mathrm{x}$ is continuous and $\varphi$ is of bounded variation on the interval $[\mathrm{a}, \mathrm{b}]$.

Lemma 1 [7]: If $\mathrm{x}$ is Stieltjes integrable on [a, b] with respect to a function $\varphi$ bounded variation, then,

$\left.\left|\int_{a}^{b} x(t) d \varphi(t)\right| \leq \int_{a}^{b} \mid x(t) d \stackrel{t}{V} \varphi\right)$,

Moreover, the following inequality holds,

$\left|\int_{a}^{b} x(t) d \varphi(t)\right| \leq \sup _{a \leq t \leq b}|x(t)|\left(V_{a}^{t} \varphi\right)$.

Lemma 2 27]: Let $x_{1}, x_{2}$ be Stieltjes integrable functions on the interval $[\mathrm{a}, \mathrm{b}]$ with respect to a nondecreasing function $\varphi$ and such that $x_{1}(t) \leq x_{2}(t)$, for $t \in[a, b]$. Then,

$$
\begin{aligned}
& \int_{a}^{b} x_{1}(t) d \varphi(t) \leq \int_{a}^{b} x_{2}(t) d \varphi(t) \text { In what follows, we } \\
& \int_{a}^{b} x(s) d_{s} g(t, s) \begin{array}{l}
\text { will also consider the Stieltjes integral of the } \\
\text { form } \\
\text { Where } g:[a, b] \times[a, b] \rightarrow R \text { and the symbol } d_{s}
\end{array}
\end{aligned}
$$
indicates the integrations with respect to $s$. The details concerning the integral of this type will be given later.

Now, let us assume that $f:[a, b] \times R \rightarrow R$ is a given function. Then, to every real function $\mathrm{x}$ defined $[\mathrm{a}, \mathrm{b}]$, we may assign the function $(F x)(t)=f(t, x(t)), t \in[a, b]$. The operator $\mathrm{F}$ defined in this way is called superposition operator generated by the function $f(t, x)$.

The properties of the superposition operator may be found in[2]. For our further purposes, we shall need the following result concerning the behavior of the superposition operator $\mathrm{F}$ on the space $C[a, b]$ consisting of all continuous functions acting from the interval $[\mathrm{a}, \mathrm{b}]$ into $\mathrm{R}$ and furnished with the standard maximum norm $\|x\|=\max \{|x(t)|: t \in[a, b]\} \quad[2]$

Lemma 3[7]: Let $\mathrm{F}$ be the superposition operator generated by the function $f:[a, b] \times R \rightarrow R$. Then, $\mathrm{F}$ transforms the space $\mathrm{C}[\mathrm{a}, \mathrm{b}]$ into itself and is continuous if and only if the function $\mathrm{f}$ is continuous on the set $[a, b] \times R$. 
Further, let us assume that $\mathrm{x}$ is a real function defined on [a,b]. Then, by $w(x, \varepsilon)$, we denote the modulus of continuity of the function $\mathrm{x}$, i.e.

$w(x, \varepsilon)=\sup \{|x(t)-x(s)|: t, s \in[a, b],|t-s| \leq \varepsilon\}$.

If $p(t, s)=p:[a, b] \times[c, d] \rightarrow R$, then, the modulus of continuity of the function $s \rightarrow p(t, s)$ on the interval [c, d ] for a fixed $t \in[a, b]$, is defined as

$$
w(p(t, .), \varepsilon)=\sup \{|p(t, u)-p(t, v)|: u, v \in[c, d],|u-v| \leq \varepsilon\} .
$$

In the similar way, we define the modulus $w(p(., s), \varepsilon)$.

3. Properties of nonlinear integral operators of mixed type integral equations

Let I be a bounded and closed interval in R. For convenience, we assume that $I=[0, b]$

In this section, we will investigate the nonlinear integral operator of mixed type of integral equations having the form

$(G x)(t)=\int_{0}^{t} v(s, x(s)) d_{s} g(t, s)+\int_{0}^{b} k(t, s) h(s, x(s)) d_{s} g(t, s), t \in I$

In our further considerations, we shall always assume that the following conditions are satisfied.

I ) $g: I \times I \rightarrow R$ and for every $t_{1}, t_{2} \in I$, such that $t_{1}<t_{2}$, the function $s \rightarrow g\left(t_{2}, s\right)-g\left(t_{1}, s\right)$ is non-decreasing on the interval I. II) $g(0, s)=0$, for any $s \in I$.

III) $v, h: I \times R \rightarrow R$ are continuous functions such that there exist a continuous functions $a, c: I \rightarrow I$ and a non-decreasing functions $\varphi, \psi: R_{+} \rightarrow R_{+}$, for which the following inequalities holds, $|v(t, x)| \leq a(t) \varphi(|x|)$, and $|h(t, x)| \leq c(t) \psi(|x|)$,for

$t \in I$ and $x \in R$.

And there exist continuous function $k: R_{+} \times R_{+} \rightarrow R$, and the following inequality is holds $|k(t, s)| \leq L$, where $L=\max \{k(t, s): t, s \in I\}<\infty$.

Remark 1 : observe that assumptions (I ) and (II) imply that the function $s \rightarrow g(t, s)$ is nondecreasing on the interval $\mathrm{I}$, for any fixed $t \in I$ [3]. Indeed, putting (I), $t_{2}=t, t_{1}=0$ and keeping in mind (II), we obtain the desired conclusion. 
From this observation, it follows immediately, for every $t \in I$, the function $s \rightarrow g(t, s)$ is of bounded variation on I. such a condition was assumed in [3], but we showed above that it is superfluous.

Now, we prove a few results about the properties of the integral operator $\mathrm{G}$ defined by (1). We start with the following theorem.

Theorem 1 : Assume that assumptions (I)-(III) are satisfied. Then, for every function $x \in C(I)$ the function $G x$ is of bounded variation on I.

Proof : observe that taking into account our assumptions, in view of lemma (3) and Remark (1), we infer that the integral operators $G$ defined by (1) is well-defined on the space $C(I)$.

Next, fix a partition $0=t_{0}<t_{1}<\ldots<t_{n}=b$ of the interval I. Then, in view of lemma (1) and Remark (1), we get

$$
\begin{aligned}
& \sum_{i=1}^{n}\left|(G x)\left(t_{i}\right)-(G x)\left(t_{i-1}\right)\right| \leq \sum_{i=1}^{n}\left|\int_{0}^{t_{i}} v(s, x(s)) d_{s} g\left(t_{i}, s\right)-\int_{0}^{t_{i-1}} v(s, x(s)) d_{s} g\left(t_{i}, s\right)\right|+ \\
& +\sum_{i=1}^{n}\left|\int_{0}^{t_{i-1}} v(s, x(s)) d_{s} g\left(t_{i}, s\right)-\int_{0}^{t_{i-1}} v(s, x(s)) d_{s} g\left(t_{i-1}, s\right)\right|+ \\
& +\sum_{i=1}^{n} \int_{0}^{b} k\left(t_{i}, s\right) h(s, x(s)) d_{s} g\left(t_{i}, s\right)-\int_{0}^{b} k\left(t_{i-1}, s\right) h(s, x(s)) d_{s} g\left(t_{i}, s\right) \mid+ \\
& +\sum_{i=1}^{n} \int_{0}^{b} k\left(t_{i-1}, s\right) h(s, x(s)) d_{s} g\left(t_{i}, s\right)-\int_{0}^{b} k\left(t_{i-1}, s\right) h(s, x(s)) d_{s} g\left(t_{i-1}, s\right) \mid \\
& =\sum_{i=1}^{n}\left|\int_{i-1}^{t_{i}} v(s, x(s)) d_{s} g\left(t_{i}, s\right)\right|+\sum_{i=1}^{n}\left|\int_{0}^{t_{i-1}} \int_{0}^{1} v(s, x(s)) d_{s}\left[g\left(t_{i}, s\right)-g\left(t_{i-1}, s\right)\right]\right|+ \\
& +\sum_{i=1}^{n} \int_{0}^{b}\left[k\left(t_{i}, s\right)-k\left(t_{i-1}, s\right)\right] h(s, x(s)) d_{s} g\left(t_{i}, s\right)+ \\
& +\sum_{i=1}^{n}\left|\int_{0}^{b} k\left(t_{i-1}, s\right) h(s, x(s)) d_{s}\left[g\left(t_{i}, s\right)-g\left(t_{i-1}, s\right)\right]\right|
\end{aligned}
$$




$$
\begin{aligned}
& \leq \sum_{i=1}^{n} \underset{t_{i-1}}{t_{i}} \mid v(s, x(s)) d_{s}\left(\underset{u=t_{i-1}}{\stackrel{s}{V}} g\left(t_{i}, u\right)\right)+ \\
& +\sum_{i=1}^{n} \int_{0}^{t_{i-1}} \mid v(s, x(s)) d_{s}\left(\underset{u=0}{S}\left(g\left(t_{i}, u\right)-g\left(t_{i-1}, u\right)\right)\right)+ \\
& +\sum_{i=1}^{n} \int_{0}^{b}\left|k\left(t_{i}, s\right)-k\left(t_{i-1}, s\right) \| h(s, x(s))\right| d_{s}\left(\stackrel{s}{V} g\left(t_{i}, u\right)\right)+ \\
& +\sum_{i=1}^{n} \int_{0}^{b} \mid k\left(t_{i-1}, s\right) \| h(s, x(s)) d_{s}\left(\underset{u=0}{\stackrel{s}{V}}\left(g\left(t_{i}, u\right)-g\left(t_{i-1}, u\right)\right)\right)
\end{aligned}
$$

Consequently, keeping in mind the above estimate, lemma (2), and Remark (1) we obtain

$$
\begin{aligned}
& \sum_{i=1}^{n}\left|(G x)\left(t_{i}\right)-(G x)\left(t_{i-1}\right)\right| \leq \sum_{i=1}^{n} \int_{t_{i-1}}^{t_{i}}|v(s, x(s))| d_{s} g\left(t_{i}, s\right)+ \\
& +\sum_{i=1}^{n} \int_{0}^{t_{i}}|v(s, x(s))| d_{s}\left(g\left(t_{i}, s\right)-g\left(t_{i-1}, s\right)\right)+ \\
& +\sum_{i=1}^{n} \int_{0}^{b}\left|k\left(t_{i}, s\right)-k\left(t_{i-1}, s\right) \| h(s, x(s))\right| d_{s} g(b, s)+ \\
& +\sum_{i=1}^{n} \int_{0}^{b}\left|k\left(t_{i-1}, s\right) \| h(s, x(s))\right| d_{s}\left(g\left(t_{i}, s\right)-g\left(t_{i-1}, s\right)\right) \\
& \leq\|a\| \varphi(\|x\|)\left\{\sum_{i=1}^{n} \int_{t_{i-1}}^{t_{i}} d_{s} g\left(t_{i}, s\right)+\sum_{i=1}^{n} \int_{0}^{t_{i}} d_{s}\left[g\left(t_{i}, s\right)-g\left(t_{i-1}, s\right)\right]\right\}+ \\
& +\int_{0}^{b}\left(\sum_{i=1}^{n}\left|k\left(t_{i}, s\right)-k\left(t_{i-1}, s\right) \| h(s, x(s))\right|\right) d_{s} g(b, s)+ \\
& +L\|c\| \psi(\|x\|) \sum_{i=1}^{n} \int_{0}^{b} d_{s}\left[g\left(t_{i}, s\right)-g\left(t_{i-1}, s\right)\right] \\
& =\|a\| \varphi(\|x\|)\{g(b, b)-g(b, 0)\}+ \\
& +\|c\| \psi(\|x\|) L\{[g(b, b)-g(0, b)]-[g(b, 0)-g(0,0)]\}<\infty .
\end{aligned}
$$

This complete the proof.

For our further purpose, we will need the following lemma.

Lemma 4 : Assume that the function $g(t, s)=g: I \times I \rightarrow R$ satisfies assumption (i).Then, for every $s_{1}, s_{2} \in I$, such that $s_{1}<s_{2}$, the function $t \rightarrow g\left(t, s_{2}\right)-g\left(t, s_{1}\right)$ is nondecreasing on the interval I [3].

Now, we prove a generalization of the result contained in theorem (1). 
Theorem 2: Suppose assumptions of theorem 1 are satisfied. Moreover, assume that the values of the functions $v, h: I \times R \rightarrow R_{+}$. Then, for every function $x \in C(I)$, the function $G x$ is nondecreasing on the interval I.

Proof: Fix arbitrarily $t_{1}, t_{2} \in I, t_{1}<t_{2}$. Next, let us take an arbitrary $0=s_{0}<s_{1}<\ldots<n_{n}=b$ of the on the interval $\mathrm{I}=[\mathrm{a}, \mathrm{b}]$ and choose arbitrarily points $c_{i} \in\left[s_{i-1}, s_{i}\right],(i=1,2, \ldots, n)$. Then, keeping in mind lemma (4), we deduced the following inequalities,

$$
\begin{aligned}
& \sum_{i=1}^{n} v\left(c_{i}, x\left(c_{i}\right)\right)\left[g\left(t_{1}, s_{i}\right)-g\left(t_{1}, s_{i-1}\right)\right]+ \\
& +\sum_{i=1}^{n} k\left(t_{i}, c_{i}\right) h\left(c_{i}, x\left(c_{i}\right)\right)\left[g\left(t_{1}, s_{i}\right)-g\left(t_{1}, s_{i-1}\right)\right] \\
& \leq \sum_{i=1}^{n} v\left(c_{i}, x\left(c_{i}\right)\right)\left[g\left(t_{2}, s_{i}\right)-g\left(t_{2}, s_{i-1}\right)\right]+ \\
& +\sum_{i=1}^{n} k\left(t_{i}, c_{i}\right) h\left(c_{i}, x\left(c_{i}\right)\right)\left[g\left(t_{2}, s_{i}\right)-g\left(t_{2}, s_{i-1}\right)\right] \\
& \leq \sum_{i=1}^{n} v\left(c_{i}, x\left(c_{i}\right)\right)\left[g\left(t_{2}, s_{i}\right)-g\left(t_{2}, s_{i-1}\right)\right]+\int_{t_{1}}^{t_{2}} v(s, x(s)) d_{s} g\left(t_{2}, s\right)+ \\
& +\sum_{i=1}^{n} k\left(t_{2}, c_{i}\right) h\left(c_{i}, x\left(c_{i}\right)\right)\left[g\left(t_{2}, s_{i}\right)-g\left(t_{2}, s_{i-1}\right)\right]
\end{aligned}
$$

This yields the inequality,

Since $\int_{t_{1}}^{t_{2}} v(s, x(s)) d_{s} g\left(t_{2}, s\right) \geq 0$

$\int_{0}^{t_{1}} v(s, x(s)) d_{s} g\left(t_{1}, s\right)+\int_{0}^{b} k\left(t_{1}, s\right) h(s, x(s)) d_{s} g\left(t_{1}, s\right)$

$\leq \int_{0}^{t_{1}} v(s, x(s)) d_{s} g\left(t_{2}, s\right)+\int_{t_{1}}^{t_{2}} v(s, x(s)) d_{s} g\left(t_{2}, s\right)+$

$+\int_{0}^{b} k\left(t_{2}, s\right) h(s, x(s)) d_{s} g\left(t_{2}, s\right)$

$\leq \int_{0}^{t_{2}} v(s, x(s)) d_{s} g\left(t_{2}, s\right)+\int_{0}^{b} k\left(t_{2}, s\right) h(s, x(s)) d_{s} g\left(t_{2}, s\right)$.

and the proof is complete. 
Our next result is connected with the continuity of the operator G.

Theorem 3 : Suppose that there are satisfied the assumptions of theorem 1. Moreover, assume that the function $s \rightarrow g(b, s)$ is continuous on I and the function $t \rightarrow g(t, s)$ is continuous on I, for any fixed $s \in I$. Then, the operator $\mathrm{G}$ transforms continuously the space C(I) into itself.

Proof : First of all let us notice that the operator $\mathrm{G}$ can be written as the compositions $G=T \circ F+M \circ N$ of the superposition operators

$$
(F x)(t)=v(s, x(s)) \quad \text { and } \quad(N x)(t)=k(t, s) h(s, x(s))
$$

and the linear Volterra- Stieltjes and Fredholm-Stieltjes operators $\mathrm{T}$ and $\mathrm{M}$, defined by the formula

$$
(T x)(t)=\int_{0}^{t} x(s) d_{s} g(t, s) \quad \text { and } \quad(M x)(t)=\int_{0}^{b} x(s) d_{s} g(t, s)
$$

Further, let us notice that in view of lemma (3) and assumptions (iii) the superposition operators $\mathrm{F}$ and $\mathrm{N}$ continuously the space $\mathrm{C}(\mathrm{I})$ into itself.

Thus, it is sufficient to show that the operators $\mathrm{T}$ and $\mathrm{M}$ acts continuously from $\mathrm{C}(\mathrm{I})$ into $\mathrm{C}(\mathrm{I})$.

To prove this, let us fix $x \in C(I)$ and a number $\varepsilon>0$. Next, take arbitrarily $t_{1}, t_{2} \in I, t_{1}<t_{2}$ such that $\left|t_{2}-t_{1}\right| \leq \in$.Then, applying lemma (1) and (2) we have

$$
\begin{aligned}
& \mid\left(T x \left(\left(t_{2}\right)-\left(T x \left(\left(t_{1}\right)|\leq| \int_{0}^{t_{2}} x(s) d_{s} g\left(t_{2}, s\right)-\int_{0}^{t_{1}} x(s) d_{s} g\left(t_{2}, s\right) \mid+\right.\right.\right.\right. \\
& +\left|\int_{0}^{t_{1}} x(s) d_{s} g\left(t_{2}, s\right)-\int_{0}^{t_{1}} x(s) d_{s} g\left(t_{1}, s\right)\right| \\
& \leq\left|\int_{t_{1}}^{t_{2}} x(s) d_{s} g\left(t_{2}, s\right)\right|+\left|\int_{0}^{t_{1}} x(s) d_{s}\left[g\left(t_{2}, s\right)-g\left(t_{1}, s\right)\right]\right| \\
& \leq|| x \mid\left\{\left[g\left(t_{2}, t_{2}\right)-g\left(t_{2}, t_{1}\right)\right]+\left|g\left(t_{2}, t_{1}\right)-g\left(t_{1}, t_{1}\right)\right|+\right. \\
& \left.+\left|g\left(t_{2}, 0\right)-g\left(t_{1}, 0\right)\right|\right\}
\end{aligned}
$$


and on the same way, we get $\left|(M x)\left(t_{2}\right)-(M x)\left(t_{1}\right)\right| \leq\|x\|\left\{\left|g\left(t_{2}, b\right)-g\left(t_{1}, b\right)\right|+\left|g\left(t_{2}, 0\right)-g\left(t_{1}, 0\right)\right|\right\}$

Hence, in view of Remark (1) and lemma (4), we arrive to the following estimate,

$\mid\left(T x\left(\left(t_{2}\right)-\left(T x\left(\left(t_{1}\right) \mid \leq\|x\|\left\{\left[g\left(b, t_{2}\right)-g\left(b, t_{1}\right)\right]+\left|g\left(t_{2}, t_{1}\right)-g\left(t_{1}, t_{1}\right)\right|+\right.\right.\right.\right.\right.$

$\left.+\left|g\left(t_{2}, 0\right)-g\left(t_{1}, 0\right)\right|\right\}$

$\leq\|x\|\left\{w(g(b,),. \varepsilon)+w\left(g\left(., t_{1}\right), \varepsilon\right)+w(g(., 0), \varepsilon)\right\}$,

and

$\left|(M x)\left(t_{2}\right)-(M x)\left(t_{1}\right)\right| \leq\|x\|\{w(g(., b), \varepsilon)+w(g(., 0), \varepsilon)\}$,

Where

the

$w(g(b,),. \varepsilon), w\left(g\left(., t_{1}\right), \varepsilon\right), w(g(., 0), \varepsilon)$ and $w(g(., b), \varepsilon)$

symbols

introduced in section 2 .

From (2) and (3), we conclude that $T x, M x \in C(I)$.

In order to show that $\mathrm{T}$ and $\mathrm{M}$ are continuous on the space $C(I)$ let us fix $x \in C(I)$.

Then, in view of lemma (1) and (2) we obtain

$$
\begin{aligned}
& |(T x)(t)| \leq \int_{0}^{t}|x(s)| d_{s}(\underset{u=0}{V} g(t, u)) \\
& =\|x\|[g(t, b)-g(t, 0)]
\end{aligned}
$$

and

$$
\begin{aligned}
& |(M x)(t)| \leq \int_{0}^{b}|x(s)| d_{s}(\underset{u=0}{V} g(t, u)) \\
& =\|x\|[g(t, b)-g(t, 0)]
\end{aligned}
$$

Hence, keeping in mind lemma (4) we conclude that the following estimate holds

$|(T x)(t)| \leq\|x\|[g(b, b)-g(b, 0)]$

Consequently

$\|T x\| \leq\|x\|[g(b, b)-g(b, 0)]$

and

$|(M x)(t)| \leq\|x\| \quad[g(b, b)-g(b, 0)]$

and Consequently 


$$
\|M x\| \leq\|x\|[g(b, b)-g(b, 0)]
$$

The inequalities (4), (5) shows that $\mathrm{T}$ and $\mathrm{M}$ transforms continuously the space $C(I)$ into itself. The proof is complete.

In what follows we show that the integral operators of mixed type of nonlinear integral equation $\mathrm{G}$ is also compact under suitable hypotheses.

Theorem 4 : Under the assumptions of theorem (3) the operator $\mathrm{G}$ is continuous and completely continuous ( takes bounded sets into relative compacts sets ) on the space $C(I)$.

Proof : Let us take a bounded subset $\mathrm{X}$ of the space $C(I)$ and take $x \in X$. Next, fix $\varepsilon>0$ and $t_{1}, t_{2} \in I$, such that $\left|t_{2}-t_{1}\right| \leq \in$.

Then, arguing similarly as in the proof of theorem (3), we obtain $\left|(G x)\left(t_{2}\right)-(G x)\left(t_{1}\right)\right| \leq\|a\| \varphi(\|x\|)\left\{w(g(b,),. \varepsilon)+w\left(g\left(., t_{1}\right), \varepsilon\right)+\right.$

$+w(g(., 0), \varepsilon)\}+L\|c\| \psi(\|x\|)\{w(g(., b), \varepsilon)+$

$+w(g(., 0), \varepsilon)\}$,

Hence, we infer that the functions of the set $G X$ are equicontinuous on the interval I. On other hand, for an arbitrary function $x \in X$, we have

$$
\begin{aligned}
& |(G x)(t)| \leq \int_{0}^{t}|v(s, x(s))| d_{s}\left(\underset{u=0}{V^{s}} g(t, u)\right)+ \\
& \quad+\int_{0}^{b} \mid k(t, s) \| h(s, x(s)) d_{s}\left(\stackrel{s}{V}_{u=0} g(t, u\right. \\
& =\{\|a\| \varphi(\|x\|)+L\|c\| \psi(\|x\|)\}[g(t, b)-g(t, 0)],
\end{aligned}
$$

In view of lemma (4), the above inequality yields

$|(G x)(t)| \leq\{\|a\| \varphi(\|x\|)+L|| c|| \psi(\|x\|)\}[g(b, b)-g(b, 0)]$

Consequently, we obtain

$$
\|G x\| \leq\{\|a\| \varphi(\|X\|)+L\|c\| \psi(\|X\|)\}[g(b, b)-g(b, 0)],
$$

Where we have denoted

$$
\|X\|=\sup \{\|x\|: x \in X\} \text {. }
$$

This shows that the set $G X$ is bounded in the space $C(I)$. 
Finally, linking the equicontiuity and the boundness of the set $G X$ is relatively compact in this space. We complete the proof.

\section{$\underline{\text { References }}$}

1 ) J. J.Abdul, "Introduction to integral equations with applications", New York and Basel, 1985.

2 ) J. Banas, "Some properties of Urysohn - Stieltjes integral operators", In. J.Math. and Math.Sci., vol.(21), no.(1), p(7988), 1998.

3 ) J. Banas and J. Ddronka, "Integral operators of Volterra- Stieltjes type, their properties and applications ", Math. and Com. Mod., vol.(32), p(1321-1331), 2000.

4 ) J. Caballero, B,Lopez and K. Sadarangani, "On monotonic solutions of an integral equation of Volterra type with supermum", J.Math.Anal.Appl., vol.(305), p(304-315), 2005.

5 ) C.Corduneanu, "Integral equations and applications ", Cambridge, New York, 2008.

6 ) A. N. Kolmogorov and Fomin, " Introductory real analysis ", New York, 1970.

7 ) I. P. Natanson, "Theory of functions of a real variable", New York, 1964.

8 ) B. G. Pachpatte," On a nonlinear Volterra - Fredholm integral equation ", Sar. J. of Math., vol.(4), no.(16),p(61-71), 2008.

9 ) H. C. Samuel, " Volterra - Stieltjes integral equations with linear constraints and discontinuous solutions ", Bulletin the Amer. Math Sci, vol.(81), no.(3), p(593-598), 1975.

10 ) P.T. Vaz and S.G. Deo, "On a Volterra -Stieltjes integral equations ", J. of Appl. Math. and Stoch. Anal, vol.(3), no.(3), p(171191), 1990. 